\title{
Influencia de un Programa de Actividades Artísticas - Culturales - Deportivas y Recreativas, en una Población Vulnerable, Exequiel González Cortez, Santiago - Región Metropolitana, Santiago-Chile
}

\author{
González Orb, Marcelo*, Carlos Bobadilla Araneda, Verónica Castro \\ González, Cristián Castro Molina, Natalia Osorio Vera, Francisca Roco \\ Osorio*
}

\begin{abstract}
Resumen
Estudio mixto, cuantitativo-cualitativo, cuyos objetivos se orientan a determinar las bases sobre las cuales se han de elaborar programas de intervención en poblaciones vulnerables; proponer y validar el programa que facilite recuperar espacios públicos; determinar las actividades de interés; establecer la intención de participación por edad y género; los elementos obstaculizadores, y determinar la influencia en el comportamiento conductual y social. La muestra está conformada por $n=4800$ sujetos de distintas edades y sexos; los instrumentos encuesta interés poblacional, cuestionario auto-diagnóstico drogadicción, encuesta percepción delincuencia y violencia poblacional, permiten determinar la influencia de la variable de intervención. Las conclusiones permiten señalar: que el programa impacta a los diferentes grupos etarios, tanto en la vida diaria, como en su integración; respecto a la participación por edad y género, esta se inclina al femenino, siendo fundamentalmente niños y jóvenes.
\end{abstract}

Palabras clave: Vulnerabilidad, Integración, Desarrollo social, Sociabilidad.

The impact of a programme of artistic, cultural, sports and recreation activities on a vulnerable population, Exequiel González Cortés Village, Metropolitan Region, Santiago, Chile

\begin{abstract}
Mixed quantitative-qualitative study aimed at determining the bases upon which vulnerable village intervention programmes have been designed; it proposes and validates programmes which help recover public spaces. The study also aims at identifying activities of interest, determining participation interest per age and gender, identifying hindering elements and the influence on self and social behaviour. The sample consists of $n=4800$ subjects of different age and sex. The instruments -population interest survey, self-diagnosis questionnaire on substance abuse and survey on crime and violence perception- help determine the influence of the intervention variable. The conclusions point out the idea that the programme has impact on different age groups, in their everyday lives as well as in their integration. Regarding participation per age and gender, this shows a female trend, and participation of mainly children and young people.
\end{abstract}

Key words: Vulnerability; Integration; Social Development; Socialisation.

* Doctor de la Universidad Metropolitana de Ciencias de la Educación.

Equipo del Departamento de Educación Física, Deportes y Recreación (DEFDER), Universidad Metropolitana de Ciencias de la Educación. 
Influencia de un Programa de Actividades Artísticas - Culturales - Deportivas y Recreativas, en una Población Vulnerable, Exequiel González Cortez, Santiago - Región Metropolitana, Santiago-Chile - Marcelo González Orb, Carlos Bobadilla Araneda, Verónica Castro González, Cristián Castro Molina, Natalia Osorio Vera, Francisca Roco Osorio

\section{Introducción}

La convivencia sana puede de una $u$ otra forma aportar al desarrollo de las comunidades, y/o traer la paz interna por lo menos durante su aplicación, pero se planean algunas dudas a resolver: ¿qué pasa con las problemáticas de tipo social?, ¿qué cambios se producen fruto del desarrollo de la práctica deportiva?, ¿qué pasa durante su desarrollo?, ¿qué cambios significativos se manifiestan en la población, en cuanto a hábitos de vida, fruto de su aplicación? Todas estas problemáticas de una u otra manera son materia de análisis, más aún en comunidades que experimentan o viven por lo general en violencia, las que están encubiertas y latentes, pero se han de estudiar para aportar a su erradicación.

La actividad física en sí, viene a otorgar las bases facilitadoras, pues se constituye en una excelente herramienta de oportunidad para aportar al cambio, pues facilita la igualdad, la equidad social, a la calidad de vida e integración, en especial de aquellos grupos más vulnerables de la sociedad. De tal forma que apreciar cuánto influye en el cambio de hábitos conductuales de aquellas personas pertenecientes a poblaciones de alto riesgo, constituye todo un reto.

El reto de tal manera se orienta a levantar evidencia que permita determinar cuán efectivo resulta ser un programa en enfrentar e incorporar a la sociedad a aquellas personas pertenecientes a poblaciones que, por lo general manifiestan conductas socialmente poco aceptadas por la sociedad, como son la violencia, la presencia de hábitos ligados actos violentos, o la reducción de espacios que van en contra de una vida sana, como representa ser la práctica de la actividad física como herramienta de salud y calidad de vida, es decir, a través de ella, efectuar una aproximación acerca de la forma en que el fenómeno deportivo-recreativo-cultural, viene a contribuir a dar solución o por lo menos a mitigar lo que hoy constituye el foco de mayor preocupación, no tan solo de quienes se encuentran al frente de las organizaciones gubernamentales, como es el Estado, sino la sociedad en general.

La importancia de la experiencia permite disponer de elementos objetivos que de lugar a afinar la mirada apreciando los beneficios de la presencia de la actividad física en las poblaciones vulnerables, 
determinando si. Un programa de intervención de actividades artísticas, culturales y deportivas, influye positivamente en el comportamiento social de la población.

Los objetivos del estudio, se orientan a: determinar las bases sobre la cual se han de elaborar los programas de intervención en poblaciones vulnerables; proponer un programa de actividades artísticas, culturales, deportivas y recreativas, que faciliten recuperar los espacios públicos en poblaciones vulnerables; elaborar y validar un programa de intervención a aplicar en poblaciones vulnerables; describir las actividades físicas, culturales, deportivas y recreativas de mayor interés para la comunidad; corroborar la intención de participación a partir de las variables género y edad; establecer elementos obstaculizadores y facilitadores para la práctica de la actividad física y deportes en la población; determinar la influencia de este en el comportamiento conductual y social de sus integrantes. Las hipótesis se orientan a establecer si: $\mathrm{H}_{\mathrm{I}}$, Un Programa de actividades artísticas, culturales, deportivas y recreativas, influye significativamente en el comportamiento social de los sujetos pertenecientes a poblaciones vulnerables, y si: $\mathrm{H}_{2}$, Un Programa de actividades artísticas, culturales, deportivas y recreativas, influye en el cambio conductual de sujetos pertenecientes a poblaciones vulnerables.

\section{Material y métodos}

\section{Población de estudio}

En su desarrollo participaron sujetos pertenecientes a la población Exequiel González Cortez de la comuna de Ñuñoa, de Santiago, Chile, declarada de alta vulnerabilidad, constituida por 800 casas, en las cuales habita un promedio de $n=6$ individuos, con una población aproximada a los $\mathrm{N}=4.800$ habitantes, de diferentes edades y sexos, (información aportada por la Unidad Vecinal No 35 "Exequiel González Cortez.

\section{Métodos Cuantitativos}

Estos se orientaron a determinar la causa efecto, respecto a la influencia de la variable Independiente, "Programa de Actividades Artísticas, Culturales, Deportivas y Recreativas, orientado a poblaciones 
Influencia de un Programa de Actividades Artísticas - Culturales - Deportivas y Recreativas, en una Población Vulnerable, Exequiel González Cortez, Santiago - Región Metropolitana, Santiago-Chile - Marcelo González Orb, Carlos Bobadilla Araneda, Verónica Castro González, Cristián Castro Molina, Natalia Osorio Vera, Francisca Roco Osorio

Vulnerables", de seis sesiones, de cuatro horas de duración, con frecuencia de dos veces por semana. Respecto a las variables dependientes, estas se orientan a determinar la influencia ejercida por el programa en cuanto a modificación del comportamiento social de los sujetos; cambios conductuales; nivel de adhesión por participar en el desarrollo de las actividades, en distintas edades; establecer el grado de neutralización del consumo de drogas, alcohol, recuperación del espacio público (calle, plaza) para una sana convivencia.

Respecto a las variables intervinientes controladas, destacan, el control del nivel de alcoholismo y drogadicción, número de participantes en las actividades; la determinación del interés de los participantes por las actividades desarrolladas, el uso de espacios públicos.

En el desarrollo del proceso indagativo, se utilizaron diversos instrumentos: encuesta de interés poblacional (a efecto de determinar los intereses artísticos, culturales, deportivos y recreativos de los participantes), para facilitar la elaboración del programa; cuestionario auto-diagnóstico orientado a determinar el nivel de conciencia, respecto a los riesgos ante el consumo de alcohol (Audit) sea de forma responsable o de abuso en niveles de riesgo, según género y edad; encuesta detección consumo de drogas (conductas reñidas con la calidad de vida y la salud), a objeto de determinar la cantidad de individuos que presentan problemas relacionados con el consumo de sustancias psicoactivas; encuesta de percepción delincuencia y violencia poblacional, todo ello orientado a establecer comparaciones una vez aplicada la variable de intervención.

\section{Métodos cualitativos}

Estos están encaminados a recabar información que permite enriquecer la información cuantitativa, aspectos imposibles de obtener con medios convencionales de indagación; entre ellos destacan el uso de fichas de observación, orientadas a llevar registro de conductas de los grupos durante el desarrollo de las actividades realizadas, teniendo como objetivos de utilización, determinar conductas de riesgo, protectoras y de resiliencia presentes o ausentes en los participantes; instrumento de registro anecdótico, 
el cual expresa las observaciones realizadas durante las sesiones, estando relacionados con la determinación del cumplimiento de los objetivos y la aplicación de las actividades, además de especificar acontecimientos de ocurrencia durante su desarrollo. Este proceder permitió registrar hechos significativos, de ocurrencia en cuanto a comportamiento y cambio de conducta de los participantes, dando lugar a describir situaciones manifestadas durante su desarrollo, es decir, pesquisar los cambios producidos en cuanto al comportamiento social de las personas durante el desarrollo de las actividades.

Descripción de la variable independiente, "Programa de Actividades Artísticas, Culturales, Deportivas y Recreativas, orientado a poblaciones Vulnerables", conformado por un total de 23 actividades: artísticas (5) $21 \%$, culturales (3) I3\%, deportivas (6) 26\%, recreativas (9) 40\%, total $100 \%$. Teniendo como objetivos la mejora de la integración social, fomentar aspectos como: sentido de autosuperación, de cohesión a nivel grupal, del potenciar habilidades personales, que faciliten la inclusión social, aumentar la autoestima, estimular el autocontrol, confianza e identidad, fomenten habilidades sociales, desarrollen valores como cooperación, compañerismo, respeto, empatía y el sentido de compromiso, mitiguen conductas negativas como egoísmo, envidia, baja tolerancia, frustración, entre otras.

\section{Aspectos contemplados en su elaboración}

En su preparación se tuvieron presentes elementos que de una u otra forma intervienen en los participantes de la población, entre los cuales destacan: las características y composición del grupo a intervenir, edad, sexo e intereses de los destinatarios, selección de actividades, determinación de procesos de transferencia, metodologías utilizadas, infraestructura, el apoyo de la familia, nivel de compromiso de las autoridades, permisos de uso de vías públicas, relación profesoresparticipantes, horario de desarrollo, recursos humanos, económicos y materiales disponibles, determinación de mecanismos de supervisión y control, nivel de compromiso y participación de los sujetos, duración del programa y sesiones, horarios de desarrollo, estación del año, frecuencia de las sesiones, condiciones de seguridad, recursos técnicos, determinación de mecanismos de evaluación, reuniones 
Influencia de un Programa de Actividades Artísticas - Culturales - Deportivas y Recreativas, en una Población Vulnerable, Exequiel González Cortez, Santiago - Región Metropolitana, Santiago-Chile - Marcelo González Orb, Carlos Bobadilla Araneda, Verónica Castro González, Cristián Castro Molina, Natalia Osorio Vera, Francisca Roco Osorio

de coordinación, contacto con los residentes, experiencia equipo interventor, facilidades otorgadas por el entorno, características sociales, espacios físicos, cantidad y calidad, elementos de seguridad, días de ejecución, apoyo de organizaciones afines, aspectos de singular importancia que forman parte de las bases y sostén de la propuesta.

En relación a las variables en estudio, la variable independiente programa de intervención, fue sometida al protocolo de juicio de experto, por profesionales con vasta y reconocida experiencia e integrantes de la propia población, de distintas edades y género.

Las variables dependientes están orientadas a determinar la influencia respecto al cambio de comportamiento social de los sujetos de la población, a establecer el aumento de participación en actividades artísticas, culturales, deportivas y recreativas, en distintas edades de la población en estudio; a neutralizar la drogadicción y recuperar el espacio público, calle, plaza, para una sana convivencia. Las variables intervinientes están directamente relacionadas a la naturaleza del estudio, es decir, disminución de alcoholismo y drogadicción; aumento de participantes en actividades y determinar el interés de los participantes por las actividades desarrolladas.

\section{Resultados}

En función a los resultados obtenidos, es posible determinar que: respecto a las bases sobre las cuales se elaboró el programa de intervención, estas cumplen plenamente con los objetivos propuestos, manifestado tanto por el interés de participación, activa (participación) y pasiva (observacional), de los sujetos de la población, como también por la adquisición de hábitos de comportamiento social, como nivel de adhesión alcanzado durante su desarrollo.

En relación a cambios de comportamiento social, se puede inferir que, aplicada la variable independiente, el comportamiento de los sujetos fue modificado con el transcurso de la aplicación del programa, manifestado por la apertura de sus integrantes, revelado por invitaciones a sus hogares, lo cual no es habitual, como por una mayor integración, demostraciones de mayor tolerancia y menor agresividad. 
En referencia al aumento en la participación en actividades artísticas, culturales, deportivas y recreativas, por edades se puede señalar que el aumento fue significativo, de $\mathrm{n}=100$, sujetos iniciales, equivalentes al 2,08\% de los participantes, mayoritariamente niños, aumentó explosivamente a más de $n=2000$, es decir, existió un aumento de más de un 4I,I6\%, respecto al total poblacional, $\mathrm{N}=4800$ sujetos, teniendo presente que al final de la intervención, existió una presencia activa de distintos grupos etarios, de edades fluctuantes, entre los 3 años a adultos mayores, es decir, personas con edades de más de 65 años.

En cuanto a la participación por género es posible señalar que las mujeres participaron más activamente que los hombres, con más del 70\% de adherentes, lo cual viene a comprobar la apertura participativa del género femenino.

En cuanto a las preferencias por actividades de la comunidad, se puede inferir que en las actividades artísticas un 53\% se inclina por manualidades, seguida, por baile con un $24 \%$ y cantar con un $8 \%$, ver gráfico ${ }^{\circ} \mathrm{I}$, Preferencias actividades artísticas.

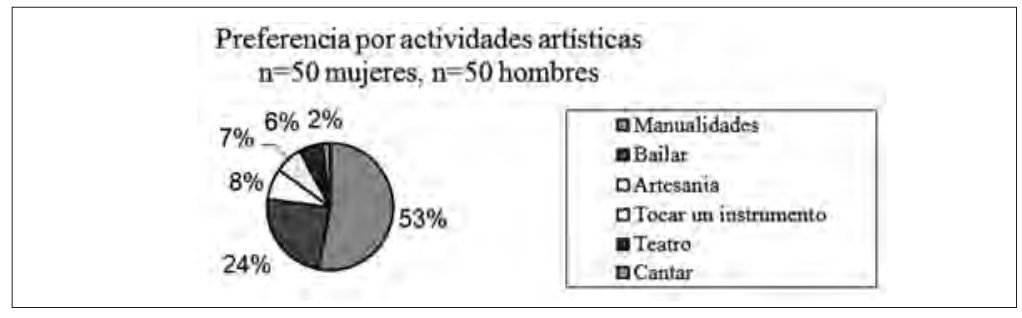

En cuanto a la adhesión a nivel cultural, destacan las preferencia de práctica y aprendizaje de bailes chilenos con un 38\% de aceptaciones; ver gráfico $n^{\circ} 2$, preferencia bailes chilenos.

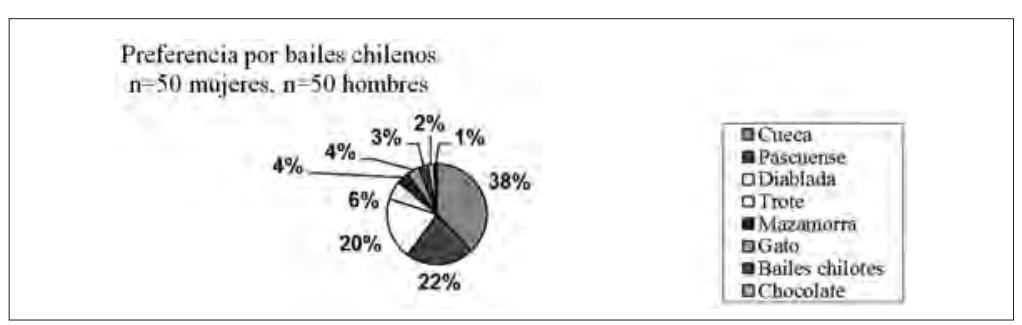


Influencia de un Programa de Actividades Artísticas - Culturales - Deportivas y Recreativas, en una Población Vulnerable, Exequiel González Cortez, Santiago - Región Metropolitana, Santiago-Chile - Marcelo González Orb, Carlos Bobadilla Araneda, Verónica Castro González, Cristián Castro Molina, Natalia Osorio Vera, Francisca Roco Osorio

En cuanto a preferencias deportivas, estas se inclinan preferentemente por fútbol con un $36 \%$, seguida por escalada con un $16 \%$ y gimnasia aeróbica, con un $10 \%$, ver gráfico $\mathrm{n}^{\circ} 3$, preferencias deportivas.

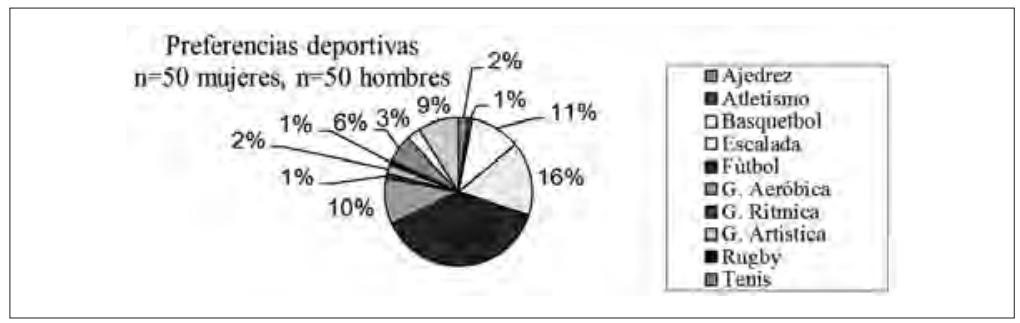

En cuanto a la participación por género y edad, es posible señalar que en ellas, participaron mayoritariamente los niños (50\%), seguida por mujeres $40 \%$ y en menos número los hombres (I0\%).

En relación a la identificación de elementos obstaculizadores, es posible señalar que estos se relacionaron, en un principio, a la falta de comunicación e inseguridad, por el grupo poblacional.

En referencia a los agentes facilitadores, es posible de destacar el factor motivacional, el querer desarrollar las actividades, aspecto que adquiere singular importancia, pues en él descansa el éxito del programa.

En relación a la recuperación de los espacios públicos, aspecto de singular importancia, se puede señalar que el programa cumplió plenamente con su objetivo, ello manifestado por el desplazamiento de personas que, antes de la intervención, los ocupaban en actividades reñidas con las buenas costumbres y la salud, como son el caso del consumo de drogas y/o alcohol en la vía pública (calles y plazas y recintos deportivos).

Como hecho destacable, es posible de mencionar, el desplazamiento de quienes deseaban adquirir sustancias psicotrópicas, los propios participantes, durante el desarrollo de las actividades, impedían su adquisición, alejando a quienes procuraban ingresar para su adquisición. 


\section{Análisis Cualitativo}

\section{Actividades relacionadas con el comportamiento social}

Al respecto se debe destacar que los sujetos al inicio de la aplicación de la variable independiente, demostraban conductas poco sociables, como agresividad, desmotivación, desconfianza, se presentan roces entre participantes, se insultan, se manifiesta la transgresión de reglas, se observa escaso autocontrol de los participantes en las actividades. Con el transcurso de la aplicación, esto va declinando, es decir, a medida que se desarrolla, se refleja un aumento de participantes, se efectúa un mayor acercamiento entre monitores y participantes, creándose ambientes aptos para la sana convivencia, sin presencia de flagelos como consumo de alcohol y drogas, la participación de la familia, niños, padres y abuelos, se manifiesta con respeto, con tolerancia y comunicación entre los pobladores.

Por otra parte, las observaciones aportan evidencias que indican que el grupo poblacional, en sus distintos segmentos etarios, demuestran cohesión y participación, siendo posible señalar que las actividades contempladas en su desarrollo influyeron positivamente en el comportamiento social y conductual, especialmente en los niños y adultos, demostrándose más abiertos, menos agresivos, y dispuestos a compartir e interactuar entre estos.

En relación al comportamiento social, el cambio es significativo; mientras al principio muestran dudas ante su eventual participación, por la desconfianza al ver la presencia de gente desconocida, dudas al desconocer el objeto de la intervención, observando desde el interior de sus casas, pero a medida que evoluciona el programa, se van integrando.

Se debe destacar que la presencia policial, trae al principio consigo, reacción de rechazo y desconfianza, en la medida que transcurre el programa, se va apreciando mayor participación y sana convivencia.

En cuanto al comportamiento conductual, al inicio los participantes se muestran temerosos y desconfiados, no respetan a los profesores y transgreden reglas; al término de la intervención, se agradece la 
Influencia de un Programa de Actividades Artísticas - Culturales - Deportivas y Recreativas, en una Población Vulnerable, Exequiel González Cortez, Santiago - Región Metropolitana, Santiago-Chile - Marcelo González Orb, Carlos Bobadilla Araneda, Verónica Castro González, Cristián Castro Molina, Natalia Osorio Vera, Francisca Roco Osorio

preocupación mostrada, se manifiestan expresiones emotivas de llanto por el término de este.

\section{Análisis Global}

Considerando los resultados, es posible inferir que el programa aplicado impactó en la vida de los pobladores, ello, determinado por las observaciones, el registro sistemático de observaciones de proceso y la opinión de los participantes, lo cual da cuenta de la influencia del programa en los diferentes grupos etarios, por edad y género. Esto se ve reflejado a través de una mayor integración de las personas, en el apoyo prestado durante el desarrollo de las actividades, en el colaborar desinteresadamente en cierres de calles, en instalar materiales, con el fin de dar continuidad y por el éxito de las mismas.

La disposición de los sujetos, los que en un comienzo se mostraban temerosos, desconfiados, reacios de participar, dio paso a la generación de lazos afectivos, de confianzas mutuas, espacios de sana convivencia, de franqueza, de tolerancia mutua empatía por parte de los participantes.

Se ha de destacar la aceptación de decisiones, por parte de los participantes, aspecto que por lo general es materia de preocupación en este tipo de escenarios, pero el espacio ganado con respeto, de profesores y participantes, constituyó una constante ascendente, es decir, aquellos que demostraron una actitud hostil al principio, asumieron el desafío, ante la práctica de la actividad física, aceptando normas de comportamiento, adquiriendo y manifestando el compromiso comunitario, de vinculación social, de cohesión y de cooperación, siendo la clave, el apoyarse los unos con los otros.

Finalmente en relación a los resultados obtenidos, es posible señalar que se observó mayor disposición de los sujetos por participar masiva y activamente, en calles, plazoletas, y pequeños recintos deportivos, ganando el espacio público.

Se impuso la generación de valores de respeto, tolerancia, empatía, beneficios de desarrollo de crecimiento personal, de socialización 
e integración comunitaria entre otros, cedieron en pro de un crecimiento pleno e integral.

\section{Discusión}

Los resultados obtenidos y contrastados con lo formulado en los contextos teóricos, permiten señalar respecto a la exclusión y la desigualdad, que estos ceden ante la aplicación de programas de corte artístico, cultural y deportivo.

La inestabilidad social, concuerda con lo planteado en el modelo teórico, respecto a la necesidad de crear y entregar oportunidades, más allá de lo laboral, lo cual otorga satisfacción, sentido de protección a los individuos clasificados como vulnerables, elementos que permiten resguardar la integridad humana, siendo mediadores de flagelos presentes en las poblaciones vulnerables, lo que permite actuar como anticipadores ante el comportamiento delictivo, del consumo de sustancias psicoactivas, de alcohol, y disminuyendo el impacto de factores de riesgo.

Por otra parte, los resultados concuerdan con lo planteado por diversos autores, en lo que respecta a que la aplicación de buenas habilidades, producen reacciones en cadena de carácter positivo, que relaciones personales seguras, elevan la autoestima, lo cual constituye una oportunidad para modificar el contexto social y el cambiar el patrón de conductas del grupo en el cual se está inmerso.

En función a la experiencia desarrollada, es posible determinar que en la actualidad, se carece de oportunidades reales de integración, fomentando con ello la discriminación.

La falta de espacios de expresión pública, se ve reflejada en el comportamiento de los individuos, el no poder expresarse libremente, los reprime contra sus pares, lo que desencadena la falta de sentido social.

Se debe de destacar que se observa falta de sensibilidad por atender a los grupos vulnerables, por lo cual surge la necesidad de establecer instancias donde los individuos recuperen su identidad, como seres 
Influencia de un Programa de Actividades Artísticas - Culturales - Deportivas y Recreativas, en una Población Vulnerable, Exequiel González Cortez, Santiago - Región Metropolitana, Santiago-Chile - Marcelo González Orb, Carlos Bobadilla Araneda, Verónica Castro González, Cristián Castro Molina, Natalia Osorio Vera, Francisca Roco Osorio

sociables, en donde puedan relacionarse, mejorar sus capacidades físicas, sociales y personales.

\section{Conclusiones}

I. Respecto a las bases sobre las que se elaboraron las actividades, es posible señalar que las dimensiones contempladas son apropiadas.

2. En cuanto a la intención de participación, a partir de las variables género y edad, la intención de participación es mayoritaria, teniendo en cuenta que cuando se otorgan las oportunidades para su desarrollo, aumenta el interés de participación tanto en hombres como en mujeres, sin importar edades.

3. En relación a los elementos obstaculizadores y facilitadores, estos están directamente relacionados a aspectos de difusión, familiarización con el entorno social, a la desconfianza, con el contar con recursos adecuados, humanos, económicos, tecnológicos, materiales e infraestructura.

4. El interés y la motivación de los sujetos, se ven afectados cuando no cumplen con las expectativas del grupo poblacional, por lo que es necesario aplicar instrumentos que permitan captar la atención e interés de los sujetos.

5. Los resultados permiten determinar que el programa influye positivamente en el grupo poblacional, manifestado por el nivel de convocatoria y de participación final de la población, la cual fue masiva.

6. Es posible señalar que el programa facilita la integración social entre los integrantes del conjunto poblacional, reflejado ello en el comportamiento y la actitud de sus semejantes, al transcurrir de la integración, al ganar espacios públicos, al surgimiento de nuevas relaciones sociales, sin distinción de edad y género.

7. En cuanto a su diseño, este cumple con los objetivos propuestos, en cuanto establece la iniciativa de autosuperación y cohesión, potencia las habilidades personales a través de la inclusión social, eleva la autoestima, autocontrol, confianza e identidad de los integrantes, fomenta las habilidades sociales a través del trabajo en equipo, desarrolla valores, incentiva la cooperación, el compañerismo, respeto, empatía, compromiso, mitiga aspectos 
negativos de comportamiento social como egoísmos, envidia, baja tolerancia a la frustración, entre otras.

8. El programa aplicado logra cambios significativos en el comportamiento social de los grupos vulnerables, otorgando una oportunidad a aquellos sujetos que han sido injustamente caracterizados como grupos de conflicto social.

A modo de síntesis, cabría preguntarse: ¿La sociedad otorga las oportunidades para salir de esta situación? ¿Otorga los espacios adecuados para desarrollar actividades deportivas-culturales? ¿Otorga recursos económicos para el fomento de las actividades, con personas debidamente calificadas para ello?

Finalmente se debe señalar que la pobreza es un problema de todos, en tal sentido todos han de estar comprometidos con ella; para erradicarla, hay que tener voluntad, el llamado es asumir este compromiso como profesionales de la educación y como sociedad en su conjunto, para superarla.

\section{Bibliografía}

Anelli. (2004). Factores Protectores Sociales. Santiago Chile: MINEDUC.

E. Balibrea Melero, J.A. Santos Ortega, I. Lerma Montero (2000). “Actividad Física, Inserción y Jóvenes socialmente desfavorecidos: aspectos fundamentales de la experiencia” Valencia, España.

Central Council of Physical Recreation (Consejo Central de Recreación Física) (Reino Unido) (2002). Everybody Wins: Sport and Social Inclusion (Todos Ganamos: Deporte e Inclusión Social), Central Council of Physical Recreation (Consejo Central de Recreación Física): Londres.

Fabre, J. y A. Hillmer (I998). "Common Ground: The Place of Sport in Development" (Terreno común: El lugar del deporte en el desarrollo), documento presentado en el Foro Olímpico Internacional para el Desarrollo, Kuala Lumpur, 9-I0 de septiembre de 1998.

Lalkaka, R. (1999). "The Role of Sporting Goods Manufacture in Economic Development" ("El papel de la fabricación de bienes deportivos en el desarrollo económico”), estudio preparado por el Programa de Desarrollo de Naciones Unidas, presentado en el Foro Olímpico Internacional de Desarrollo, junio de I999. 
Influencia de un Programa de Actividades Artísticas - Culturales - Deportivas y Recreativas, en una Población Vulnerable, Exequiel González Cortez, Santiago - Región Metropolitana, Santiago-Chile - Marcelo González Orb, Carlos Bobadilla Araneda, Verónica Castro González, Cristián Castro Molina, Natalia Osorio Vera, Francisca Roco Osorio

PNUD (2002). Informe de Desarrollo Humano, PNUD: Nueva York.

PNUMA (1992). Agenda 2I: La Declaración de Río, PNUMA.

Perona, Nélida y Silvia Robin (2000). Desocupación y vulnerabilidad. Un estudio de caso en el Barrio Parque Sur. Rosario, en Anuario del Departamento de Ciencias de la Comunicación, Año 1999/00, Volumen V, Escuela de Comunicación Social. Facultad de Ciencia Política y Relaciones Internacionales. U.N.R.

UNICEF (1999). Educación para la Paz en UNICEF, Serie de Documentos de Trabajo (Departamento de Educación), julio de 1999.

UNICEF (1999). Manual de Aplicación de la Convención sobre los Derechos del Niño, UNICEF: Nueva York.

UNICEF (2002). Los niños afectados por los conflictos armados: Las acciones del UNICEF, UNICEF: Nueva York.

UNODC (2002). Sport: Using Sport for Drug Abuse Prevention (Deporte: Utilizando el Deporte para Evitar el Abuso de Drogas), UNODC: Nueva York.

VNU (200I). Below the Waterline of Public Visibility (Bajo la línea de flotación de la visibilidad pública), informe de la Mesa Redonda de Voluntariado y Desarrollo Social en La Haya, Países Bajos, 29 y 30 noviembre 2000 .

VNU (200I). On Volunteering and Social Development (Sobre Voluntariado y Desarrollo Social), Reunión del Grupo de Trabajo de Expertos de VNUS.

\section{URL}

"Concepto de Violencia" [Consultado el I4 de mayo de 2010 desde http:/ / pisaal.blogia.com/2010/04040I-concepto-de-violencia.php] Santiago, Chile.

"Declaración Universal de los Derechos Humanos" [Consultado el I4 de mayo de 2010 desde http://www.cinu.org.mx/onu/documentos/dudh.htm] Santiago, Chile.

"El amor en familia" [Consultado el I4 de mayo de 2010 desde http://www. aciprensa.com/Familia/amarfami.htm]. Santiago, Chile.

"Grupos Vulnerables" [Consultado el I4 de mayo de 2010 desde http:// archivos.diputados.gob.mx/Centros_Estudio/Cesop/Eje_tematico/d_ gvulnerables.htm]. Santiago, Chile.

"Vulnerabilidad y Adaptación al cambio climático". [Consultado el I4 de 
mayo de 2010 desde http://www.met.igp.gob.pe/impactos/vulne. html]. Santiago, Chile.

Ficha de Protección social [Consultado el I4 de mayo de 2010 desde http:/ / www.fichaproteccionsocial.cl/fps/fps2.html]. Santiago, Chile.

Vulnerabilidad en una Zona en crisis: El Nyragongo [Consultado el I4 de mayo de 2010 desde http://www.unep.org/geo/geo3/spanish/498. html]. Santiago. Chile

Concepto de amenaza, vulnerabilidad y riesgo [Consultado el I4 de Mayo de 2010 desde http://www.tdr.cesca.es/TESIS_UPC/AVAILABLE/ TDX-04I6I02-075520//04Capitulo2.PDF Pág.8]. Santiago, Chile.

Vulnerabilidad y Exclusión social. Una propuesta metodológica para el estudio de condiciones de vida en los hogares [Consultado el I4 de mayo de 2010 desde http://www.ubiobio.cl/cps/ponencia/doc/pI5.4.html]. Santiago, Chile. 